\title{
Exposer le patrimoine de la Retirade: la pédagogie au croisement des logiques sociales et institutionnelles. Étude de cas à Argelès-sur-Mer
}

\author{
Virginie Soulier, Université de Perpignan Via Domitia \\ virginie@virginiesoulier.com
}

Résumé Soixante-quinze ans après la Guerre civile espagnole, le Centre d'interprétation et de documentation sur 1'Exil et la Retirade (CIDER) d'Argelès-sur-Mer présente l'histoire de ce passé traumatique. Rendre compte de l'enfermement de plus de 200000 Républicains espagnols dans un camp de barbelés soulève de nombreux enjeux patrimoniaux. Cet exil forcé d'un demimillion de réfugiés représente l'un des exodes européens les plus importants du siècle dernier. Il touche encore de nombreuses personnes et demeure en résonance avec l'actualité. Le présent article vise à rendre compte du projet muséographique du CIDER. Les logiques sociales et institutionnelles s'affrontent entre les moments d'émergence, de construction et de valorisation dans le processus de patrimonialisation, mais elles se recouvrent dans la perspective pédagogique de l'exposition permanente.

Mots clés: Muséologie; éducation non formelle; immigration; patrimoine; memorial; Guerre d'Espagne; citoyenneté.

\section{Introduction}

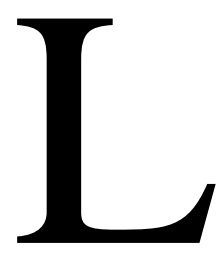

a migration forcée des Républicains espagnols de 1939, nommée la Retirada ${ }^{1}$, est l'un des exodes européens les plus importants du siècle dernier qui a été enfermé dans le silence jusqu'au tournant de l'an 2000. Peu muséalisée, mais présente dans les mémoires des exilés et de leurs descendants, cette période contemporaine soulève de nombreux enjeux patrimoniaux.

À la frontière franco-ibérique, les associations de mémoire et les élus locaux participent à l'invention du patrimoine de cette immigration et de ces lieux d'internement. Le camp d'Argelès-sur-Mer a été le premier et l'un des plus grands camps de réfugiés espagnols qui fuyaient le franquisme. Il a fermé en 1941. Le Centre d'interprétation et de documentation sur

\footnotetext{
${ }^{1}$ Le mot « Retirada » signifie « retraite » de l'armée républicaine en catalan et castillan.
} 
l'exode et la Retirade (CIDER) de cette ville, que nous nommerons «Mémorial » a ouvert en 2014. Il présente l'histoire de la Retirade et de son camp d'internement.

L'article rend compte des perspectives communicationnelles de l'exposition permanente du Mémorial d'Argelès-sur-Mer au sein du processus de patrimonialisation de la Retirade. Comment cette période historique encore douloureuse et conflictuelle est muséalisée? L'enquête met au jour les enjeux mémoriels et historiques, ainsi que le modèle de transmission mis en œuvre dans la démarche de production de l'exposition du Mémorial.

\section{Problématique}

Rodriguez (2012) et Cuesta (2012) constatent que plus de 70 ans après la fin du conflit armé, aucun musée national de la Guerre civile ou de l'histoire contemporaine de l'Espagne n'a vu le jour. Les programmes de patrimonialisation sur la dictature ont du mal à aboutir. La disparition des derniers témoins risque d'engendrer une «démémoire» (Rodriguez, 2012, p. 269). La transposition muséale de cette Guerre demeure dispersée, en Espagne ou ailleurs, et rencontre de nombreux défis à l'égard de la «mémoire hégémonique », construite et véhiculée par l'État franquiste (Leizaola, 2007, p. 488).

Dans le cadre de nos enquêtes de terrain, nous observons en Catalogne un mouvement d'actions mémorielles de la Retirade qui débute en 1977 avec la création de la fondation Antonio Machado et qui connaît ensuite une plus grande ampleur en 1999 avec le $60^{\mathrm{e}}$ anniversaire de l'exode. Au tournant de l'an 2000, le procédé de mémoration se traduit par une patrimonialisation des traces de la Retirade à la frontière franco-ibérique. Des stèles commémoratives sont érigées. Les chemins de l'exode sont balisés. Des monuments placés aux abords des anciens camps et de la frontière sont installés. Les lieux qui ont servi d'hébergement aux réfugiés sont muséalisés, comme la Maison du patrimoine de St-Laurent de Cerdans et la Maternité d'Elne. Des témoins 
viennent spontanément déposer leurs mémoires dans ces équipements patrimoniaux. Des collectes d'objets et de récits de vie sont notamment mises en place par des associations de mémoire.

Dans le contexte des préparatifs du $60^{\mathrm{e}}$ anniversaire est née l'association des Fils et filles de Républicains espagnols et enfants de l'exode, plus connue sous l'acronyme FFREEE. Elle a pour mission de rassembler et de soutenir les enfants des Républicains, de collecter et de valoriser des témoignages. De plus, FFREEE est à l'origine du projet du Mémorial de la ville. Non seulement ses locaux se situent au Mémorial mais, en plus, elle garde un rôle important dans les actions éducatives, mémorielles et expositionnelles. Le Mémorial est un centre d'interprétation et de documentation géré par le conseil municipal de la ville.

Le patrimoine de la Retirade tel qu'il est constitué par FFREEE et la communauté locale comprend des récits de vie, des objets ayant appartenu à des citoyens, des archives municipales et départementales. Pour les habitants, le paysage est chargé de mémoires sur l'exode et l'internement. De la montagne qui marque la frontière avec l'Espagne à la mer où se situait le camp de barbelé, il ne reste plus aucune trace tangible aujourd'hui. Le parcours d'interprétation sur la Retirade d'Argelès-sur-Mer comporte le Mémorial, trois monuments commémoratifs, une plaque signalétique qui indique la limite nord de l'ancien camp sur la plage et un arbre symbolique dédié aux soixante-dix enfants internés, décédés en bas âge.

\section{Comment la Retirade est exposée?}

Notre travail consiste à comprendre la mise en exposition de cette période historique. Cette recherche s'inscrit dans le champ théorique et interdisciplinaire de la muséologie qui vise à apporter un regard analytique et réflexif sur les musées (Meunier, 2012)2.

\footnotetext{
${ }^{2}$ La recherche a été financée par le Conseil de recherches en sciences humaines du Canada (CRSH).
} 


\section{Revue de littérature et ancrages théoriques}

Il ressort de notre recension des écrits sur la patrimonialisation de la Retirade des travaux récents inscrits notamment en ethnologie et en histoire. Citons les travaux de Moulinié $(2011,2013)$ et de Rousseau (2012, 2013).

Moulinié et Sagnes se sont surtout intéressées aux mémoires des républicains espagnols et aux acteurs du patrimoine, c'est-à-dire aux actions mises en œuvre par les associations de mémoire comme étape d'émergence et de construction dans le processus de patrimonialisation (2011, 2013). Nous, nous concentrerons plus spécifiquement sur la mise en exposition de la Retirade.

L'approche historique dans les travaux de Rousseau invite surtout à la vigilance quant aux musées d'histoire et aux lieux de mémoire. Elle consiste à mettre en garde vis-à-vis des diverses formes de récupération de l'histoire. Le programme de recherche intitulé la «muséohistoire » interroge au sein du discours muséal l'articulation entre les savoirs historiques et les mémoires (Rousseau et al, 2012, 2013). La transposition muséographique de l'histoire est pensée comme un « observatoire de fabrication mémorielle » (Rousseau et al, 2012). Cette approche consiste à mettre en évidence les visions dites «falsificatrices du passé », les « oublis historiographiques » ou encore les «persistances mystificatrices » (Rousseau et al., 2012). Au-delà de ces précautions qui relèvent essentiellement des exigences scientifiques de la discipline de l'histoire, nous envisageons l'exposition selon deux ancrages théoriques en sciences de l'éducation et en sciences de l'information et de la communication. L'exposition est conceptualisée comme une situation d'apprentissage (Allard \& Boucher, 1998) et un média (Davallon, 1992, 1999). Elle est porteuse de significations. Elle est un moyen de diffusion. Elle est pensée comme un système de transmission de partage des savoirs (Schiele, 2011). Nous interrogeons le rapport au savoir tel qu'il y est instauré. 


\section{Démarche de recherche}

Notre projet vise à porter un éclairage sur le traitement muséographique de l'exposition permanente du Mémorial. L’objectif est de cerner le modèle de transmission subjacent.

Afin de délimiter notre travail, nous nous focalisons sur l'espace de production de l'exposition. Notre démarche consiste à prendre appui sur les intentions des concepteurs-muséographes qui ont présidé à la mise en exposition, puis d'analyser la perspective communicationnelle de l'exposition. Pour cela, nous avons mené des entretiens avec les responsables du Mémorial, les membres du comité de production et du comité scientifique pour qu'ils explicitent la démarche de production du Mémorial et de l'exposition permanente. Nous avons ensuite mené une analyse expographique qui consiste à identifier la logique de production de l'exposition, mais aussi à remonter aux orientations constitutives du programme expositionnel tant conceptuelles que structurelles. Pour construire notre grille d'analyse, nous nous sommes notamment appuyée sur les travaux de Chaumier (2013), Davallon (2000, 2011), Veron et Levasseur (1989), Montpetit (1996) et Poli (2003).

\section{Résultats: le programme muséographique de l'exposition permanente}

L'exposition a été réalisée avec le concours de plusieurs partenaires: le conseil municipal, l'Association FFREEE, le comité scientifique composé essentiellement d'historiens et l’entreprise de scénographie Anagram audiovisuel.

\section{1- Le parcours de visite: le rapport au savoir historique}

En premier lieu, l'organisation de l'espace correspond à une transposition du savoir historique.

L'exposition de $200 \mathrm{~m}^{2}$ porte sur la thématique de l'exode des Républicains espagnols venus en France. Elle est structurée en trois séquences: 1- 1'Espagne - pays de départ; 2- le passage de la frontière - la Retirade; 3- la France - pays d'accueil des réfugiés. La première séquence traite de 
la guerre en Espagne. Elle est articulée en quatre sections: 1- Le franquisme; 2- La Guerre civile espagnole; 3- Un conflit médiatisé; 4- Un conflit international. La deuxième séquence marque le passage de la frontière. Le côté français est caché par une cloison. Sur le modèle de la transmigration, les visiteurs doivent avancer pour découvrir ce qui les attend derrière celle-ci. Pour passer d'un pays à l'autre, le visiteur doit nécessairement passer par la frontière, symbolisée par un couloir noir, où une douche sonore fait entendre les bombardements. La troisième séquence concerne les camps en France. Elle est divisée en deux sections: 1- les camps d'internement de la France métropolitaine; et 2- Le camp d'Argelès-sur-Mer. Cette dernière zone est le cœur de l'exposition. Au centre de cette aire se trouve un îlot. Celui-ci est composé de panneaux suspendus et de caisses en bois qui font office de bancs. Ces moyens scénographiques forment un cube. Ils laissent imaginer un espace restreint au sein d'un baraquement. En ce sens, le camp est modelé sans être reproduit. Le bois sur les murs, dans cette dernière séquence, rappelle également les planches des baraquements utilisées dans la construction des camps. Ce dispositif suggère le lieu d'internement (Montpetit, 1996, p. 57). Le circuit repose ainsi sur le principe de l'évocation indicielle (Veron et Levasseur, 1989, p. 35).

L'analyse du circuit montre que le programme de l'exposition est construit selon une structure chronologique et géographique. Il restitue une certaine cohérence historique des évènements. Selon une perspective métonymique, les visiteurs parcourent le déroulement temporel de la guerre en Espagne et le déplacement des exilés vers la frontière. Ils sont ensuite confinés et se retrouvent finalement, dans l'immobilité, voire dans l'enfermement, pour explorer les conditions de vie dans les camps. Cet engagement corporel demeure une modalité d'appropriation du discours expositionnel. La conception de l'aménagement spatial repose sur cette activité de déambulation pour créer du sens. Le sens a pour support le corps du visiteur (Veron et 
Levasseur, 1989, p. 35). D’un point de vue opératoire, le parcours de visite figure le chemin de l'exil et le lieu d'internement.

En deuxième lieu, le dispositif de transmission du savoir s'appuie sur des matériaux qui ont présidé à l'élaboration du discours historique. À titre d'aide à l'interprétation en contexte expositionel, les articles de presse faisaient partie au préalable des corpus de recherche. De plus, la construction des dispositifs expositionnels s'appuie sur les outils de diffusion traditionnellement employés par les historiens et les didacticiens de l'histoire: la carte historique et la chronologie (figure 1). Les visiteurs doivent être familiers avec ce champ de connaissances pour être à même de s'approprier les informations ainsi médiatisées de manière autonome.

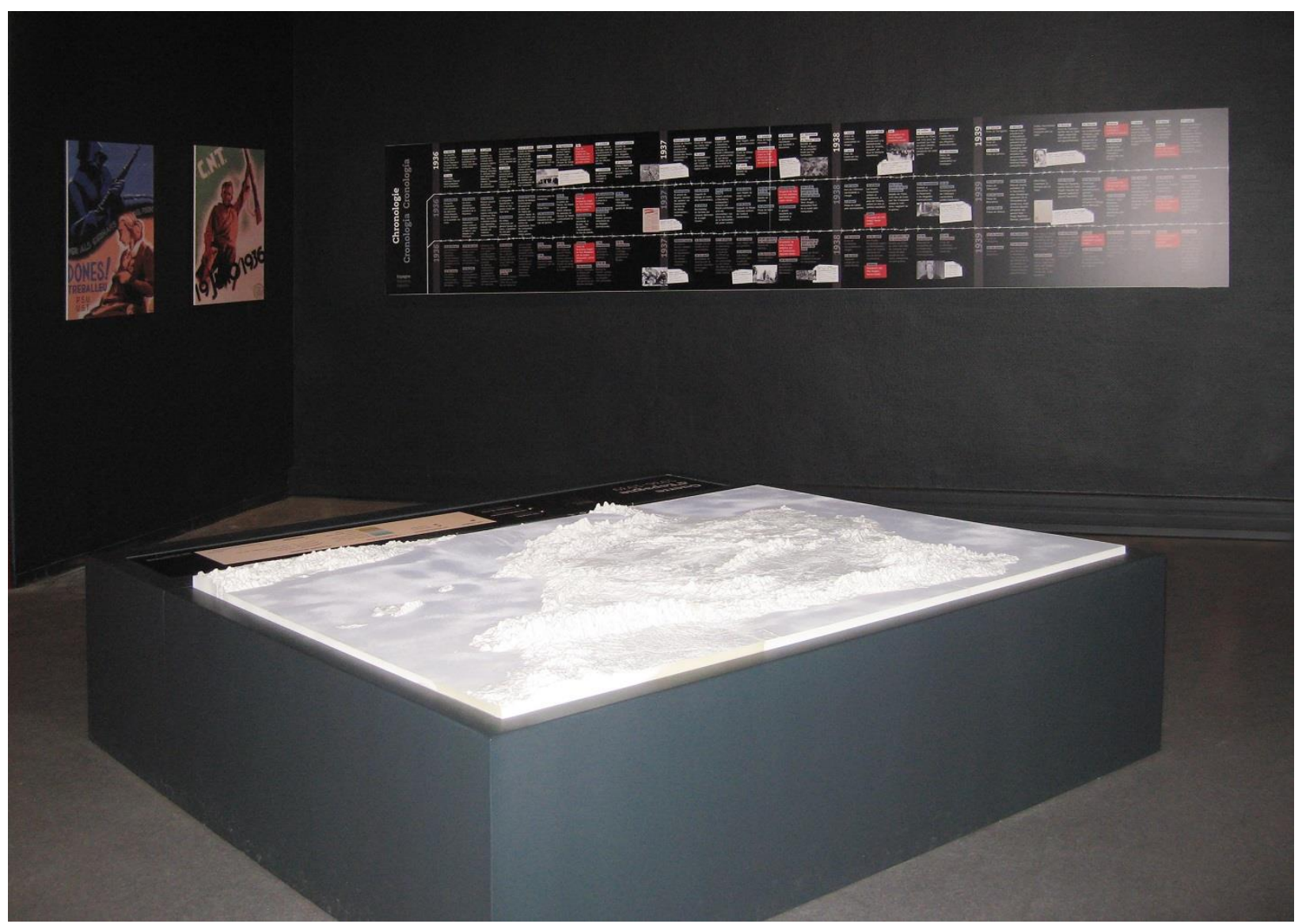

Figure 1: Carte interactive et chronologie dans la séquence 1 de l'exposition (V. Soulier, 2015) 
Enfin, l'esthétique de l'espace marque une double temporalité. Elle signifie un temps de guerre révolu. L'ambiance plastique marque aussi un régime historique présent. Elle exprime ainsi une vision contemporaine sur cette période historique.

\section{2- Un climat de guerre}

Les moyens scénographiques traduisent un climat de guerre et permettent de contextualiser cette période historique. Deux camps antagonistes ressortent de manière assez simplifiée: les Républicains espagnols qui luttent pour la démocratie et les Franquistes qui combattent pour le fascisme.

D'origine artistique, iconographique, photographique, cinématographique, journalistique et administrative, des archives historiques sont reproduites sur les panneaux et diffusées par des dispositifs multimédias à titre de documents et de témoignages. Ces sources variées sont intégrées dans l'exposition pour une appréhension de la dictature, des bombardements, de l'exil et de l'expérience concentrationnaire.

La présence systématique des articles et des photographies en noir et blanc dans chacune des unités expositionnelles, rythme le trajet lectoral et visuel des visiteurs (figure 2). La récurrence de la presse manifeste le suivi journalistique important qui est fait à cette période, ainsi que l'importance que revêt à cette période le photojournalisme. Le format des photographies et la perspective de leur image varient en fonction du positionnement des visiteurs durant leur parcours. Ce qui leur attribue un rôle d'observateur. L'environnement visuel est également construit à partir d'un traitement graphique et d'un éclairage spécifique. Des changements apparaissent entre le segment expositionnel sur l'Espagne et celui de la France. On note un basculement dans le rapport à la mort. D'une mort imminente sous les bombardements, les Espagnols affrontent ensuite une mort latente dans les camps. En bref, la scénarisation restitue un 
effet de dramatisation. Le visiteur est ainsi plongé dans la souffrance et la lutte en tant que témoin.

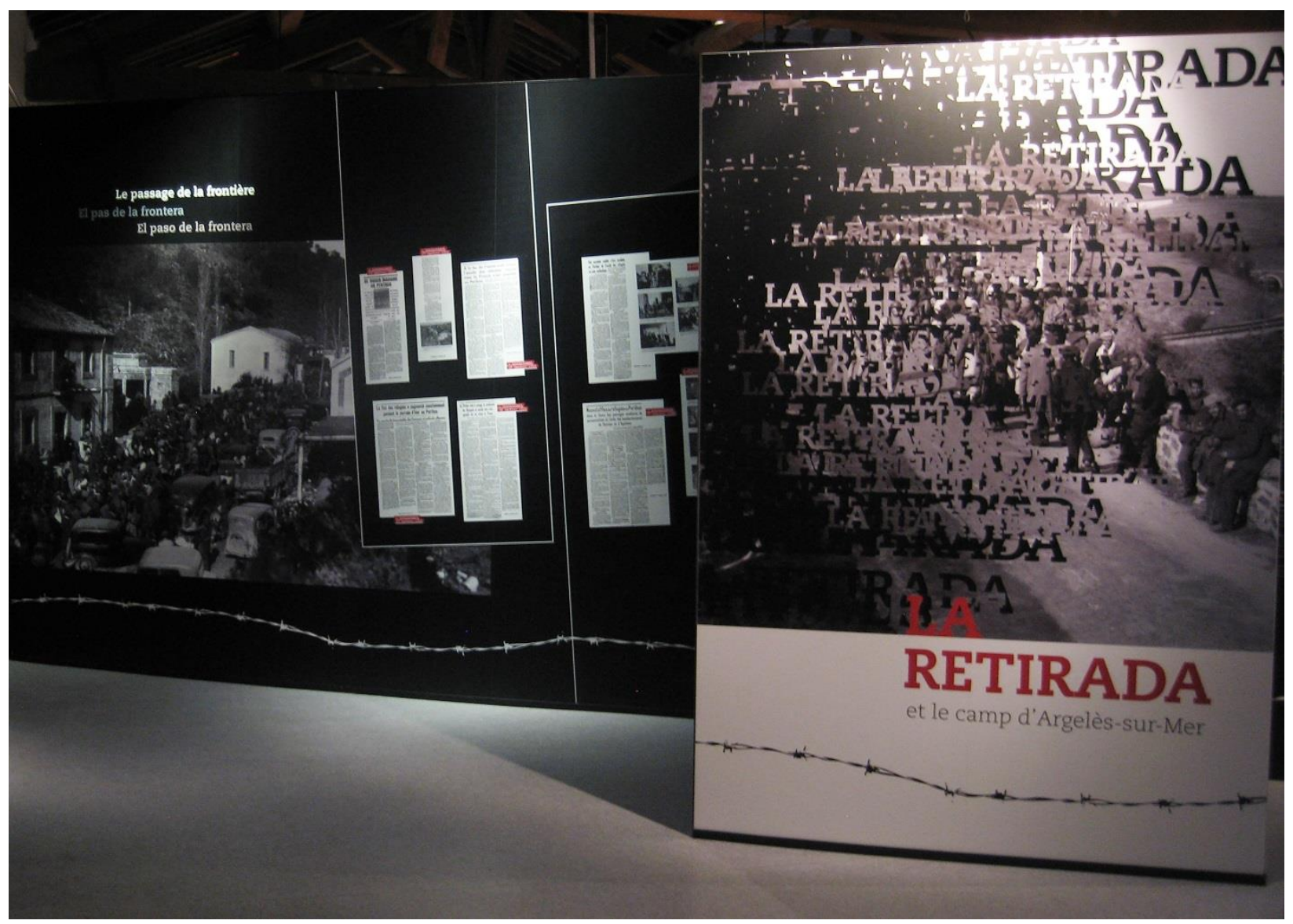

Figure 2: Exemples de photographies et d'articles de presse exposés (V. Soulier, 2015)

\section{3- Les caractéristiques de la guerre}

Dans cette ambiance de guerre omniprésente, plusieurs types de conflits sont montrés: médiatiques, idéologiques, politiques et territoriaux.

Cinq thèmes transversaux associés à la guerre apparaissent dans l'ensemble du parcours de l'exposition: le photojournalisme, les idéologies, les politiques, les territoires et la mort. Ils définissent les différentes facettes du conflit international, comme en Espagne, en France, en Angleterre, en Italie et en Allemagne. Deux buts ressortent: les guerres d'idées et les conflits territoriaux. 
En contrepartie, le régime de paix en Europe apparaît au début et à la fin de l'exposition. En premier, la résolution de «non-intervention » dans le conflit civil espagnol signée en août 1936 par les pays européens est présentée. On trouve finalement l'inscription du monolithe d'Argelèssur-Mer, érigé dans le contexte de la commémoration de 1999, qui est retranscrite sur un panneau. Elle souligne la paix et la réunification de l'Europe.

Le fil conducteur de l'exposition repose sur le travail du photojournalisme au travers des articles de presse et des photographies qui couvrent la période historique de 1931 (proclamation de la Seconde République) à 1941 (fermeture définitive du camp d'Argelès-sur-Mer).

\section{4- De la survie à la survivance: l'expérience concentrationnaire}

Dans la dernière section, les messages principaux concernent l'expérience concentrationnaire et le devoir historique de préserver cette mémoire pour les générations futures.

Plusieurs stratégies muséographiques sont utilisées pour montrer la vie dans le camp d'Argelèssur-Mer. Sur le premier mur, la projection d'une figure d'un interné, le poing gauche levé, traduit une ambiance indéfectible de lutte pour les valeurs républicaines. Un livre tactile, situé audessous de cette figure emblématique, établit le lien entre les générations du passé et du présent. Il montre, entre autres, les actions de FFREEE et du conseil municipal qui visent à retracer et à se remémorer cette période historique. Cet ensemble symbolise une lutte qui perdure. Sur le deuxième mur, une vitrine-cloison met en scène, à la manière d'un diorama, des objets disposés sur du sable avec en arrière-plan une photographie de la plage. Ces objets, sur l'ancien emplacement du camp, établissent le lien d'authenticité dans la rencontre médiatisée entre les expôts et les visiteurs. Des effets personnels de toilette sont exposés (figure 3), ainsi que les attributs les plus significatifs pour témoigner de l'exil et l'internement: une valise, une couverture et des fils de barbelés. Il s'agit d'un procédé analogique. Sur le troisième mur est 
présenté le film Camp d'Argelès, de Felip Solé, de 2009. L'intérêt de cette narration fictive est d'intégrer plusieurs témoignages d'anciens internés, mais aussi de gardes et de s'appuyer sur divers documents de journalistes ou d'amateurs de l'époque. Selon une autre approche médiatique, des témoignages oraux sont aussi à écouter. L'exposition se termine sur une sculpture réalisée par un artiste interné du camp, Jesus Lantada Buey (1939). Cette multiplication des moyens de témoigner manifeste la rupture avec le silence et marque un régime mémoriel qui vient appuyer le discours historique de l'exposition.

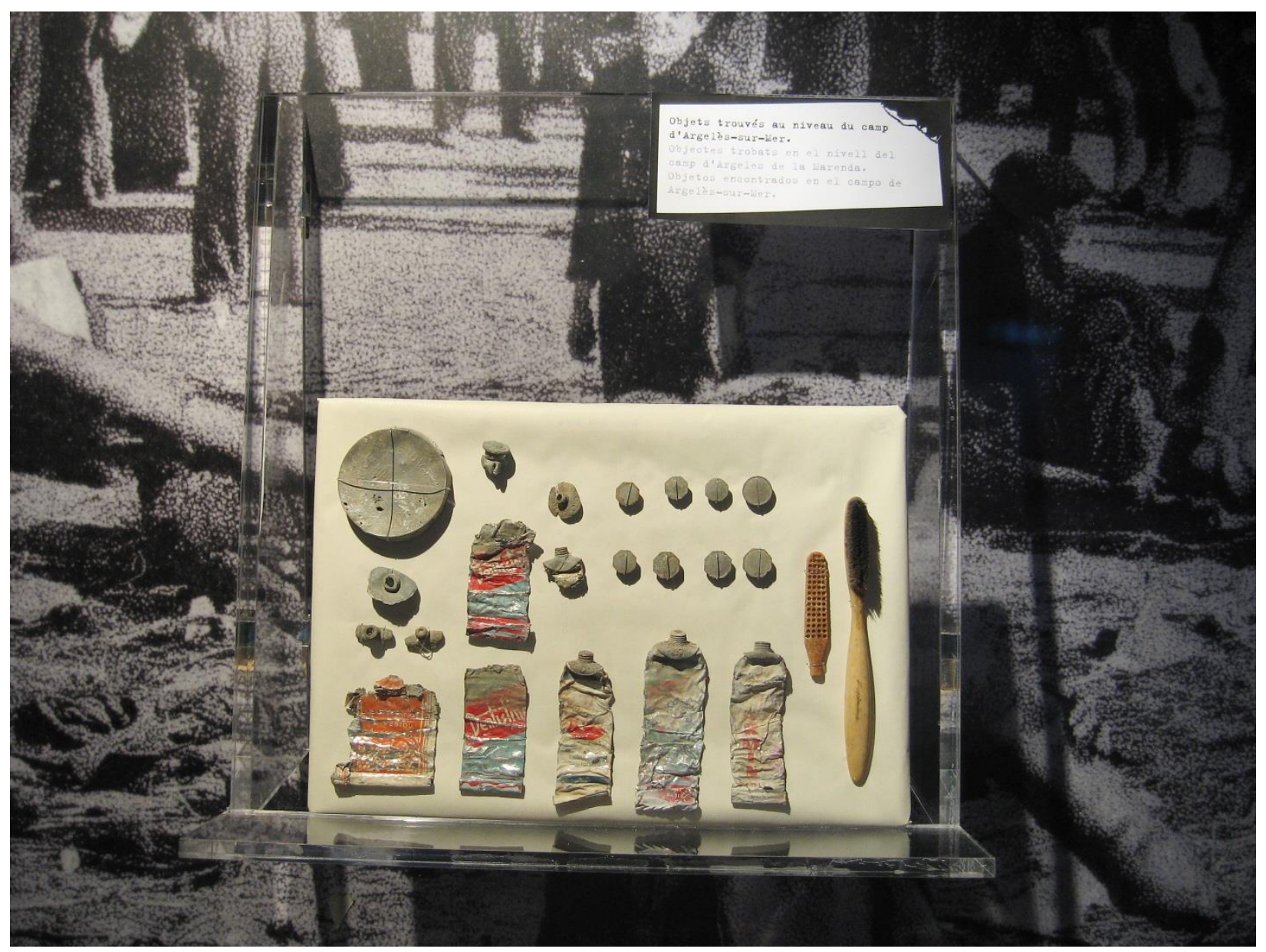

Figure 3: Effets personnels retrouvés sur la plage dans l'ancien camp (V. Soulier, 2015)

En somme, le fonctionnement de l'exposition repose sur une logique communicationnelle exogène ancrée sur la discipline de l'histoire qui se traduit par un langage didactique 
(Chaumier, 2013, p. 54-55). Ce traitement muséographique repose sur un contrat d'éducation non formelle en histoire (Schiele, 2001, p. 145). Il n'a pas pour objectif principal de développer un regard critique, une conscience sociale ou une attitude militante à l'égard des évènements actuels. Le discours de l'exposition ne tisse pas de liens avec d'autres guerres et exodes forcés qui ont lieu après la Retirade. Selon les principes de la muséographie du savoir, il communique essentiellement des informations et des connaissances historiques (Davallon, 2000). Il repose essentiellement sur un paradigme positiviste de l'histoire. Alors que Dreyfus-Armand fait partie du comité scientifique, la démarche méthodologique ne s'inscrit pas en histoire orale. Néanmoins, l'exposition propose plusieurs lectures et constructions de sens, contrairement à une situation d'apprentissage en contexte scolaire. Elle est construite sur la logique inhérente au savoir de référence qui permet ensuite d'élaborer un système de représentation culturelle (Schiele, 201, p. 144-145). Quelle est cette représentation?

\section{Discussion}

La matrice communicationnelle de cette exposition repose sur un système que nous qualifions de testimonial, car il conjugue les régimes historiques et mémoriels. Pour certains concepteursmuséographes, cette exposition ne s'appuie pas «assez» sur les témoignages des réfugiés espagnols. D'après les entretiens, les processus d'émergence et de construction du patrimoine de la Retirade reposent sur des logiques sociale et participative. Les acteurs principaux sont les membres de FFREEE. Le projet du Musée repose quant à lui sur une logique institutionnelle et politique mise en œuvre par le conseil municipal. Il apparait clairement une rupture dans le processus de patrimonialisation, tel qu'il est réalisé par la communauté d'Argelès-sur-Mer, entre la construction et la valorisation du patrimoine de la Retirade. 
Le programme muséographique a été pensé entre les associations de mémoires, les historiens et les élus locaux. Alors que plusieurs acteurs proposaient d'établir une collecte rigoureuse des témoignages oraux, les témoignages n'ont pas été enregistrés et exposés. Le Mémorial conserve de nombreux dépôts spontanés de mémoires sans qu'il n'y ait de politique d'acquisition et de collecte systématique des témoignages. Dans le cadre des entretiens avec les concepteursmuséographes, il ressort que certains voulaient «montrer les mémoires intimes des internés » pour faire ressortir les vécus singuliers et faire montre de "plusieurs sensibilités », alors que d'autres concepteurs préféraient s'appuyer sur des archives écrites d'origine administrative et journalistique pour construire une histoire plus globale. Ces derniers voulaient éviter que «les témoins se racontent ». En ce sens, le patrimoine repose sur deux logiques collectives distinctes qui forment ensemble une approche citoyenne. Le patrimoine est d'abord créé selon une logique sociale. La deuxième logique opérationnelle est d'ordre « légitimiste »(Rautenberg, 2003, p. 21). Les acteurs participent à la pérennisation et à la reconnaissance officielle du patrimoine de la Retirade, ainsi qu'à l'institutionnalisation du discours sur ce patrimoine. Le Mémorial permet ainsi de prendre en charge le patrimoine, selon une perspective de démocratisation de la culture et d'offrir un espace public, commun, gratuit et ouvert à tous. Il résulte que le patrimoine de la Retirade repose sur une construction citoyenne à la fois associative et municipale. Les deux logiques convergent vers un projet pédagogique dont le parti-pris des concepteurs pour le républicanisme est nettement affiché. Ce modèle expographique combine ainsi les approches dites républicaine et libérale de la muséologie citoyenne (Meunier et Soulier, 2010). Il s'appuie en effet sur une forte appartenance à la communauté politique et sur l'expertise d'historiens qui collaborent avec des citoyens (Meunier et Soulier, 2010, p. 323-326). Par conception de la citoyenneté, nous n'entendons pas des idéologies et des principes de gouvernement, mais plutôt 
la manière dont les acteurs se conçoivent eux-mêmes comme citoyens en tant qu'élus ou électeurs engagés. Tenant compte que ce Mémorial concerne un exode et qu'il touche des personnes issues de l'immigration qui ont été privées de leurs droits civiques et qui ont du lutter pour être reconnus et acceptés comme citoyens français, il serait intéressant de poursuivre la recherche en analysant le sentiment de citoyenneté chez les acteurs et les publics pour cerner davantage ce projet muséal.

\section{Bibliographie}

Barba, S. (2009). De la frontière aux barbelés. Les chemins de la Retirada 1939. Canet: Trabucaire.

Chaumier, S. (2013). Traité d'expologie - Les écritures de l'exposition. Paris: La Documentation française.

Cuesta, J. (2012). Les lieux de mémoire de la guerre civile à la dictature en Espagne. In Lieux de mémoire, musées d'histoire. Paris : La Documentation française.

Davallon, J. (2000). L'exposition à l'œuvre. Paris : L'Harmattan.

Davallon, J. (2011). Le pouvoir sémiotique de l'espace. Hermès. La Revue, 61(3), 38-44.

Duclos, J.-C., Poli, M.-S., Ancel, P. et collaborateurs (2014). Exposer l'histoire contemporaine : évaluation muséologique d'une exposition, spoliés! L'aryanisation économique en France 1940-1944. Paris : La Documentation française.

Idjéraoui-Ravez, L. (2012). Le témoignage exposé : du document à l'objet médiatique. Paris : L'Harmattan.

Leizaola, A. (2007). La mémoire de la guerre civile espagnole : le poids du silence. Ethnologie française, 37(3), 483-491. 
Meunier, A. et Luckerhoff, J. (2012). La muséologie, champ de théories et de pratiques. Québec : Presses de l’Université du Québec.

Meunier, A. et Soulier, V. (2010). Préfiguration du concept de muséologie citoyenne. Dans Meunier, A., J.-F. Cardin et M.-A. Éthier (sous la dir. de), Histoire, musées et éducation à la citoyenneté (pp. 309-330). Québec : MultiMondes.

Montpetit, R. (1996). Une logique d'exposition populaire: les images de la muséologie analogique. Publics et musées, 9, 55-100.

Moulinié, V. (2013). L'exode et les camps pour pays. Les descendants de Républicains espagnols en France. Ethnologie française, 43(1), 31-41.

Moulinié, V. et Sagnes S. (2011). Des exilés politiques aux vaincus magnifiques : mémoire des républicains espagnols (février 1939). Mémoires de l'immigration : vers un processus de patrimonialisation. Carcassonne : Rapport déposé au Ministère de la Culture et de la Communication/Cité Nationale de l'Histoire de l'Immigration.

Ortiz, J. (2014). La République est de Retour ! Paris : Atlantica.

Pigenet, M., Pigenet, P. et Tartakowsky, D. (2006). Espagne ou les mémoires interdites. Sociétés \& Représentations, 22(2), 148-157.

Poli, M.-S. (2003). Le texte au musée : une approche sémiotique. Paris : L’Harmattan.

Rautenberg, M. (2003). Comment s'inventent de nouveaux patrimoines: usages sociaux, pratiques institutionnelles et politiques publiques en Savoie. Culture \& Musées, 1(1), 1940.

Rodriguez, M.-C. (2012). Usages politiques des passés traumatiques en Espagne: muséohistoire de la guerre civile. Dans Rousseau, F. (sous la dir. de), Les présents des passés 
douloureux : musées d'histoire et configurations mémorielles, essais de muséohistoire (pp. 269-303). Paris : Michel Houdiard.

Schiele, B. (2001). Cinq remarques sur le rôle pédagogique de l'exposition scientifique et un commentaire sur la réforme de l'éducation. Dans Julien, L. et L. Santerre (sous la dir. de), L'apport de la culture à l'éducation. Actes du colloque de recherche : culture et communications (pp. 135-157). Montréal : Éditions Nouvelles.

Varine, H. de. (1991). L'Initiative communautaire. Mâcon: PUL.

Veron, E. et Levasseur, M. (1989). Ethnographie de l'exposition. Paris : Bibliothèque Publique d'Information. 